\title{
Expression of bone morphogenetic protein 2, 4, 5, and 7 correlates with histological activity of otosclerosis
}

\author{
PÉTER CSOMOR ${ }^{1}$, BALÁZS LIKTOR ${ }^{2}$, BÁLINT LIKTOR ${ }^{2}$, ZOLTÁN SZEKANECZ $^{3}$, \\ ISTVÁN SZIKLAI ${ }^{1} \&$ TAMÁS KAROSI ${ }^{1}$
}

\author{
${ }^{1}$ Department of Otolaryngology and Head and Neck Surgery, Medical and Health Science Center, University of Debrecen, \\ Debrecen, ${ }^{2}$ Department of Otolaryngology, Bajcsy-Zsilinszky Hospital, Budapest and ${ }^{3}$ Department of Rheumatology, \\ Medical and Health Science Center, University of Debrecen, Debrecen, Hungary
}

\begin{abstract}
Conclusion: This study is the first to establish that bone morphogenetic protein 5 (BMP5) plays a role in the pathogenesis of otosclerosis. These results confirm that elevated expression levels of BMPs, members of the transforming growth factor (TGF)- $\beta$ superfamily, contribute to the pathologically increased bone turnover in early, active stages of otosclerosis. Objectives: Otosclerosis is a complex bone remodeling disorder of the otic capsule, which might be characterized by increased expression of different types of BMPs. TGF- $\beta$ and BMP are both members of the TGF- $\beta$ superfamily and play a critical role in bone resorption and new bone formation. It has been suggested that BMP and its receptors may be involved in the pathologically increased bone turnover observed in otosclerosis. Methods: Fifty-one otosclerotic and 16 non-otosclerotic ankylotic stapes footplates were histologically analyzed: conventional hematoxylin-eosin staining and BMP2, 4, 5, and 7specific immunofluorescent assays were performed. Cortical bone fragments $(n=35)$ and incus specimens $(n=6)$ were used as negative controls. Results: Active otosclerosis $(n=39)$ was characterized by increased expression of BMP2, 4, 5, and 7 . Inactive cases of otosclerosis $(n=12)$ were characterized by negative immunoreaction for BMPs. Non-otosclerotic stapes specimens $(n=16)$ and negative controls $(n=41)$ showed negligible BMP expression. The BMP expression pattern showed a strong correlation with the histological activity of otosclerosis.
\end{abstract}

Keywords: Bone remodeling disorder, non-otosclerotic stapes fixation, stapes

\section{Introduction}

Otosclerosis is a primary disorder of bone remodeling unique to the human otic capsule and is characterized by disturbed equilibrium of bone resorption and new bone formation $[1,2]$. Otosclerotic foci usually appear in the stapes footplate and in the pericochlear region and lead to progressive conductive and/or sensorineural hearing loss as a consequence of stapes footplate fixation and pericochlear bone resorption [1]. The fissula ante fenestram often contains fibrous tissue and immature cartilage and it is a preformed anatomic structure with a predilection for both stapedial and cochlear otosclerosis [1]. Otosclerosis is strictly limited to the human otic capsule: no otosclerosis-like bone lesions have been reported outside of the ear $[1,3]$. Otosclerosis is a relatively common cause of hearing impairment in the Caucasian white population, with a prevalence of $0.3-0.4 \%$ [1]. Histological otosclerosis is much more common and it has been reported to have a prevalence of $8-11 \%$ in large unselected autopsy series [3]. According to previous reports, otosclerosis represents approximately $60 \%$ of stapes ankylosis cases leading to conductive hearing loss $[1,4]$. Several hypotheses suggested viral, inflammatory, autoimmune, metabolic, and genetic factors as possible links to otosclerosis; however, the etiopathogenesis has remained unexplained $[1,5,6]$. Correspondence: Tamás Karosi MD PhD, University of Debrecen, Medical and Health Science Center, Department of Otolaryngology and Head and Neck
Surgery, Debrecen, Nagyerdei Krt. 98, H-4032, Hungary. Tel: +36 52414763 . Fax: +36 52414 763. E-mail: karositamas@gmail.com. 
Several genetic predisposition factors for otosclerosis have been reported over recent decades $[1,6]$. According to linkage studies, familial aggregation of patients with stapes fixation typically shows an autosomal dominant inheritance with incomplete penetrance and has promoted the identification of 10 otosclerosis-associated loci (OTSC1-10) [1,6]. These loci have been mapped and linked to otosclerosis; however, specific genes and proteins have not been identified yet $[1,6]$. In most patients, otosclerosis does not follow a regular Mendelian segregation, suggesting a complex disease caused by interconnected environmental and genetic factors $[1,6]$. It has been discussed that $\mathrm{A} 1$ and $\mathrm{A} 2$ alleles of type I collagen (COL1A1/A2), different types of bone morphogenetic protein (BMP2, 4, 7), transforming growth factor- $\beta 1$ (TGF- $\beta 1$ ), CD46-type measles virus receptor, and RELN genes may also be involved in the development of otosclerosis [7-11]. However, some of these genetic associations cannot explain female dominancy, adult onset, and organ-limited pattern of otosclerosis $[1,6,7,11]$.

In the last 5 years, BMPs and their receptors (BMPRs) have been reported to contribute to the pathogenesis of otosclerosis $[8,12]$. BMPs are a group of growth factors and belong to the TGF- $\beta$ superfamily, so they also act as inflammatory cytokines $[13,14]$. At present, 20 distinct isoforms of BMP have been discovered; however, only 6 (BMPs 2-7) function as bone-specific growth factors $[13,14]$. BMP1 is not closely related to other known growth factors, since it is a matrix metalloproteinase $[13,14]$. BMPs 2-7 play an important role in the embryonic phase of endochondral bone development and later in new bone formation and repair [13,14]. BMPs 2-7 are excreted by embryonic chondrocytes, osteoid precursor cells, preosteoclasts, and inflammatory $\mathrm{T}$ cells $[13,14]$. Physiologically, BMPs act as disulfidelinked homodimers and induce bone and cartilage formation through their receptors [12-14]. Signal transduction process mediated by activated BMPRs results in mobilization and recruitment of SA (Sma and Mad related proteins) proteins $[13,14]$. Finally, SA induces the expression of TGF- $\beta 1$ [14]. BMPs and TGF- $\beta 1$ are critical regulators of bone turnover within the TGF- $\beta$ superfamily $[13,14]$. In addition, an inverse interplay has been confirmed between BMP and TGF- $\beta 1$ pathways $[13,14]$. Disruption of BMP signaling and mutations in BMPs and in their inhibitors (noggin, chordin, sclerotisin) are associated with a variety of bone diseases, including fibrodysplasia ossificans progressiva and congenital stapes fixation with multiple synostoses-synphalangism syndrome $[14,15]$. Detection of increased BMP expression in otosclerosis might support the 'embryonic cartilage reactivation hypothesis' in the pathogenesis of disease.

This study investigated the expression levels and patterns of different types of BMPs in otosclerotic and non-otosclerotic stapes footplates to elucidate the effective role of BMP association in the pathogenesis of otosclerosis.

\section{Material and methods}

\section{Patients and controls}

A total of 67 ankylotic stapes footplates $(n=67$, male $=26$, female $=41$ ) removed by stapedectomy were analyzed. The mean age of patients was 42.28 years (range 25-65 years). The diagnosis of ossicular chain fixation was based on clinical, audiometric, and tympanometric findings. Air-bone gap at $1000 \mathrm{~Hz}$ was at least $30 \mathrm{~dB}$ on pure tone audiometry. Preoperative tympanometry revealed type As tympanograms in $79.1 \%(n=53)$ and type A tympanograms in $20.9 \%$ $(n=14)$ of stapes fixation cases. Stapes fixation was clinically bilateral in 44 patients (65.7\%); however, only one stapes obtained from each patient was analyzed, because over this period, only unilateral stapes surgeries have been performed. Partially removed stapes footplates were not included in the study, because the anterior or posterior poles containing the bone lesions fixing the stapes were retained in the oval window niche. Fragmented and reconstructed footplates were not excluded. Thirty-five cortical bone fragments harvested from the external ear canal during lateral atticotomy $(n=35)$ and six incus specimens removed during canalwall-down (CWD) mastoidectomy were used as tissuespecific controls of BMP expression of stapes footplates. Cortical bones were obtained from patients with stapes ankylosis, while incus specimens originated from patients with middle ear cholesteatoma. Stapes footplates and control tissue specimens were collected from December 2009 until March 2011 (University of Debrecen, Medical and Health Science Center, Department of Otolaryngology and Head and Neck Surgery, Debrecen, Hungary; and ENT Department of Bajcsy-Zsilinszky Hospital, Budapest, Hungary). All patients gave their informed written consent to our study. The Hungarian Scientific Research Ethical Committee (ETT-TUKEB 84-227/2008-1018EKU) approved this study. The study was carried out according to the Declaration of Helsinki.

\section{$B M P$ 2, 4, and 7 specific immunofluorescent assay}

A total of 67 ankylotic stapes footplates, cortical bone fragments $(n=35)$, and incus samples $(n=6)$ were 
fixed in $10 \%(\mathrm{w} / \mathrm{v})$ buffered formaldehyde and decalcified in $0.5 \mathrm{M}$ Na-EDTA (sodium ethylenediamino-tetraacetate, $72 \mathrm{~h}, 4^{\circ} \mathrm{C}$ ) containing $0.02 \%$ $(\mathrm{w} / \mathrm{v})$ sodium azide. Then specimens were embedded in $15 \%(\mathrm{w} / \mathrm{v})$ purified gelatin $\left(24 \mathrm{~h}, 56^{\circ} \mathrm{C}\right)$ and refixed in $4 \%(\mathrm{w} / \mathrm{v})$ paraformaldehyde $\left(24 \mathrm{~h}, 20^{\circ} \mathrm{C}\right)$. Histological blocks were cryoprotected in $20 \%(\mathrm{w} / \mathrm{v})$ saccharose solution $\left(2 \mathrm{~h}, 4^{\circ} \mathrm{C}\right)$ and sectioned into $10 \mu \mathrm{m}$ slides at $-25^{\circ} \mathrm{C}$ (MNT-200, Slee, Mainz, Germany). Slides were stored in $0.1 \mathrm{M}$ PBS (phosphate-buffered saline) containing $0.03 \%(\mathrm{w} / \mathrm{v})$ sodium azide at $4^{\circ} \mathrm{C}$.

Five consecutive $10 \mu \mathrm{m}$ frozen cut sections were examined as follows. (1) Staining with hematoxylin and eosin (H\&E); and (2) BMP2, (3) BMP4, (4) BMP5, and (5) BMP7 specific immunofluorescent assays (IFAs). First, sections of each individual specimen were processed by conventional H\&E staining. The IFA series of sections were washed and protein blocked in Power Block ${ }^{\mathrm{TM}}$ Reagent (BG-BS-1310-25, Biogenex, Fremont, CA, USA). Blocked sections were incubated with 1:20 monoclonal mouse antihuman BMP2 primary antibody solution $\left(20^{\circ} \mathrm{C}, 24 \mathrm{~h}\right.$, continuous shaking) (RD-MAB2260, R\&D Systems, Minneapolis, MN, USA), with 1:20 polyclonal goat anti-human BMP2/4 primary antibody solution $\left(20^{\circ} \mathrm{C}, 24 \mathrm{~h}\right.$, continuous shaking) (RD-AF355, R\&D Systems), with 1:20 polyclonal goat antihuman BMP5 primary antibody solution $\left(20^{\circ} \mathrm{C}\right.$, $24 \mathrm{~h}$, continuous shaking) (RD-AF615, R\&D Systems), and finally with 1:20 monoclonal mouse antihuman BMP7 primary antibody solution $\left(20^{\circ} \mathrm{C}, 24 \mathrm{~h}\right.$, continuous shaking) (RD-MAB3542, R\&D Systems). Primary antibodies were washed with $0.2 \mathrm{M}$ PBS and sections were incubated with 1:200 Northern Lights ${ }^{\mathrm{TM}}$ donkey anti-goat IgG labeled by NL-493 fluorochrome $\left(20^{\circ} \mathrm{C}, 6 \mathrm{~h}\right.$, continuous shaking) (R\&D Systems) and with 1:200 Northern Lights ${ }^{\mathrm{TM}}$ donkey anti-mouse IgG labeled by NL-637 fluorochrome $\left(20^{\circ} \mathrm{C}, 6 \mathrm{~h}\right.$, continuous shaking) (R\&D Systems), respectively. All antibody incubations were performed in a sterile humid chamber. Slides were covered by UV transparent mounting medium (Vectashield ${ }^{\mathrm{TM}}$, Vector Laboratories, CA, USA). Imaging was performed under UV light with $510 \mathrm{~nm}$ and $470 \mathrm{~nm}$ wavelengths and 43-77 ms exposure time and the photographs were archived in jpeg file format (Axioskop2 MOT ${ }^{\mathrm{TM}}$, Axiovision 3.0 ${ }^{\mathrm{TM}}$, Zeiss, Jena, Germany). Individual specimens were analyzed as 10-20 centrally focused, high magnification $(\times 40)$ fields according to the BMP2, 4, 5, and 7 specific immunofluorescent reactions. Histological examinations were blinded for two independent researchers: P.C. analyzed the sections stained by H\&E, while T.K. interpreted the IFAs. The histopathological activity of otosclerosis was based on the cellularity and the structure of cement
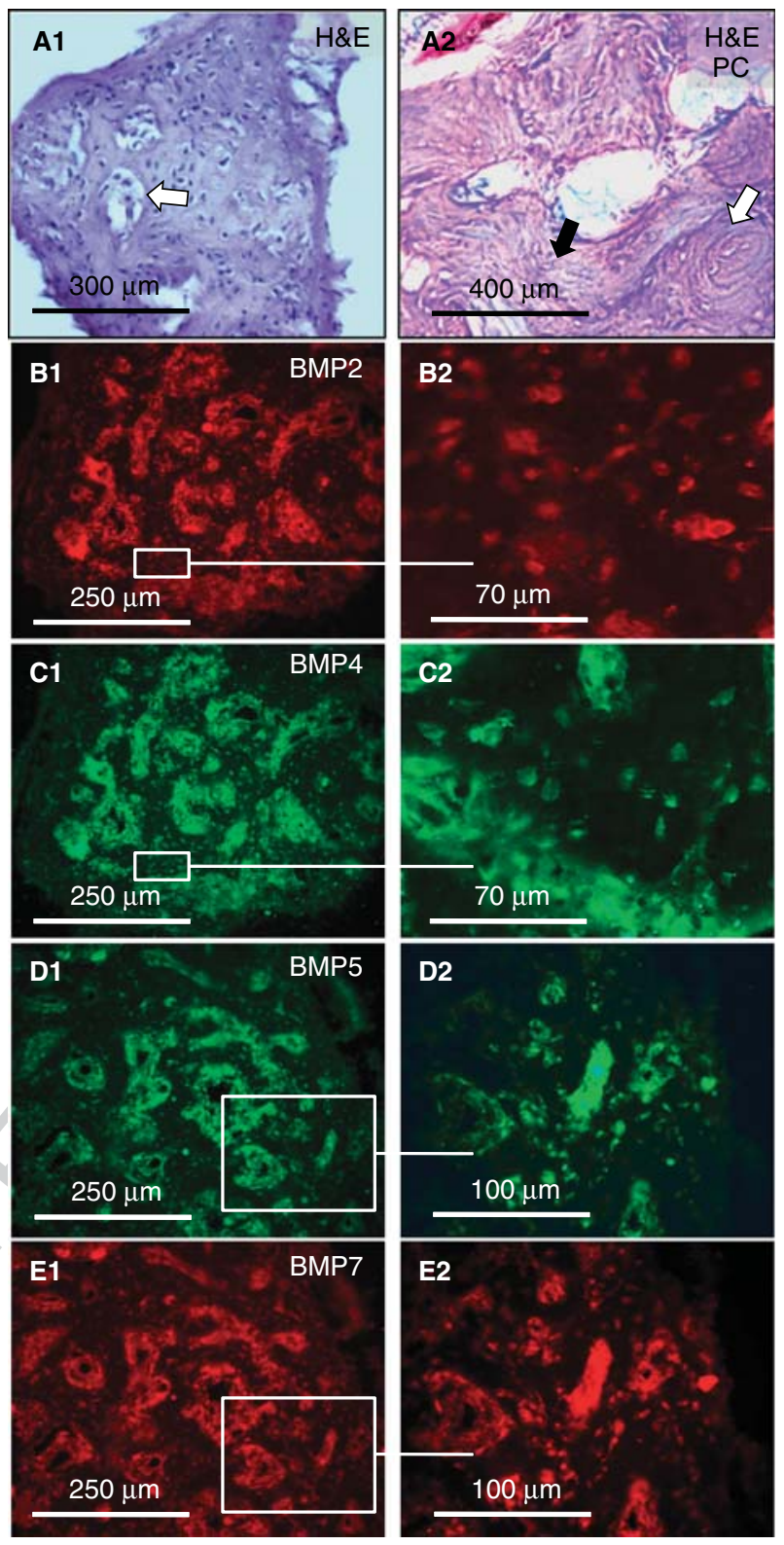

Figure 1. Immunofluorescent demonstration of BMP expression in active otosclerosis. (A1) Active focus of otosclerosis with hypercellular osteoid substance and several pseudo-vascular spaces (white arrow) (H\&E, right ear). (A2) Phase-contrast (PC) microscopic view of the previous section. The cement lines show an irregular concentric (white arrow) and woven (black arrow) pattern. Immunofluorescent micrographs with different magnification views for BMP2 (B1, B2), BMP4 (C1, C2), BMP5 (D1, D2), and BMP7 (E1, E2) display intense granular cytoplasmic immunoreactions in the lesion-forming cells.

lines by conventional H\&E staining. Since otosclerosis is a multifocal disease, the histopathological activity of a stapedial osteolytic focus is not in full correspondence with the clinical activity of otosclerosis $[1,4]$. We used the following histopathological criteria for the diagnosis of otosclerosis. (1) Active otosclerosis is displayed by wide pseudo-vascular 

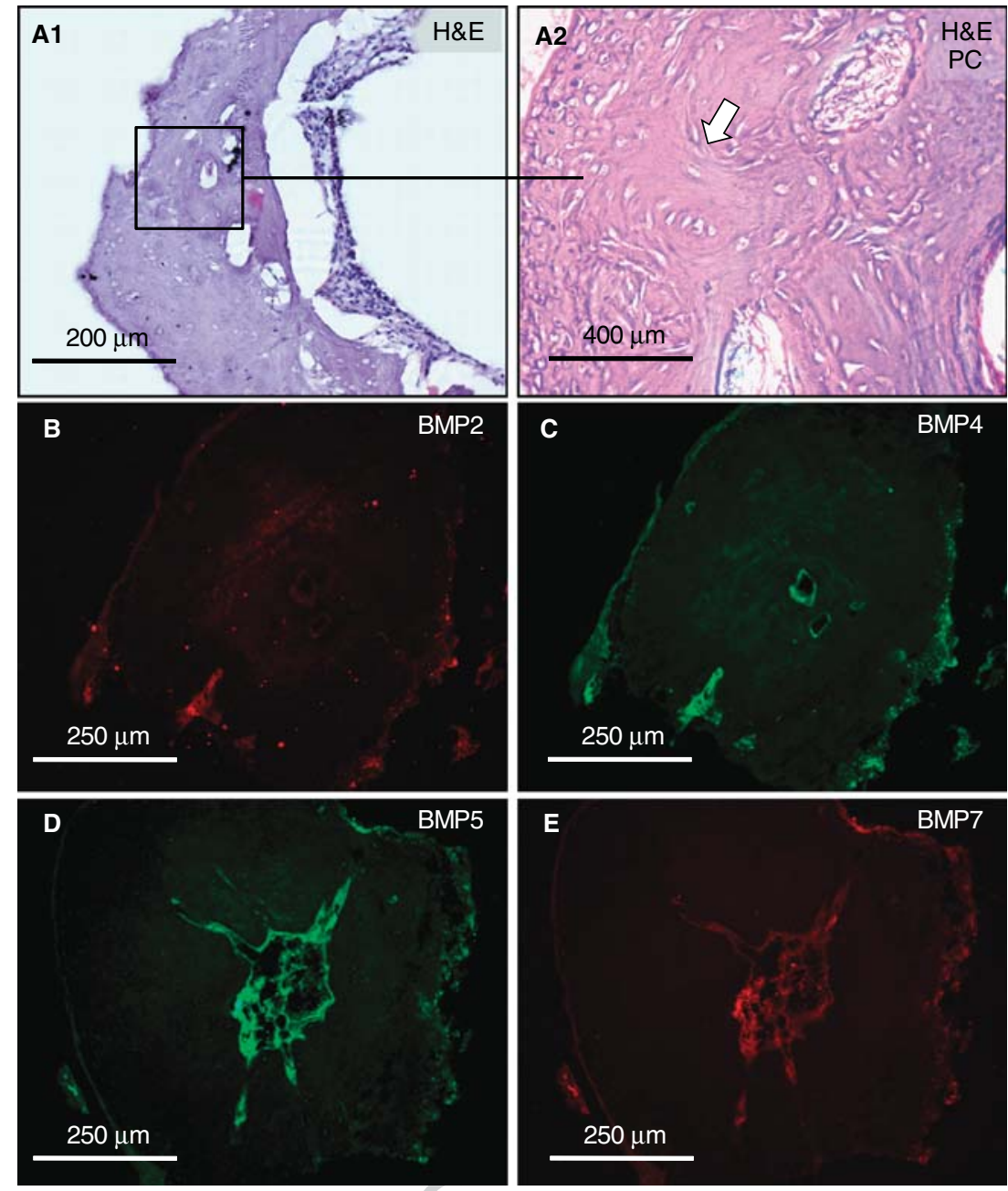

Figure 2. Immunofluorescent demonstration of BMP expression in inactive otosclerosis. (A1) Inactive otosclerotic focus at the posterior pole of the stapes footplate (H\&E, right ear). The lesion is characterized by hypocellularity and empty cellular halos. (A2) Phase-contrast (PC) microscopic view of the previous section. Cement lines show lamellar pattern (white arrow). The remnants of osteoblasts and osteoclasts (empty halo cells) show negative immunofluorescent staining for BMP2 (B), BMP4 (C), BMP5 (D), and BMP7 (E).

spaces filled with increased numbers of large, misshapen, and multinucleate osteoclasts. Cement lines show a woven pattern. (2) Inactive otosclerosis is marked by obliterated vascular spaces and resorption lacunae with decreased numbers of osteoclasts (empty halo cells). Cement lines show a lamellar pattern.

\section{Results}

Ankylotic stapes footplates $(n=67)$, cortical bone fragments $(n=35)$, and incus specimens $(n=6)$ were analyzed by BMP2, 4, 5 and 7 specific IFAs and by conventional H\&E staining, respectively. Histological diagnosis of otosclerosis was established in 51 ankylotic stapes footplates (Figures 1 and 2, Table I). Stapes footplates were involved by single and dominant otosclerotic foci. Among these specimens, foci of otosclerosis were considered to be active in 39 stapes footplates and inactive in 12 cases (Figures 1 and 2, Table I). The localization of otosclerotic foci differed among the 51 patients with manifest involvement of the stapedial footplate by otosclerosis at the anterior pole $(n=31)$, posterior pole $(n=7)$, bipolar $(n=6)$ or obliteration $(n=7)$. In the 16 non-otosclerotic stapes footplates, histologic examinations revealed annular, stapediovestibular calcification with marked thickening of the stapes footplate [4] (Figure 3, Table I). According to the clinical history and otoscopic findings of nonotosclerotic patients, tympanosclerosis due to chronic otitis media or familiar stapes fixation could be excluded.

Active otosclerosis cases were characterized by simultaneously increased expression of BMP2, 4, 5, and 7 that resulted in intense granular immunoreaction (Figure 1). Both osteoclasts and osteoblasts showed considerable positivity for different types of BMPs, 
Table I. Assessment of expression of bone morphogenetic proteins (BMPs) 2, 4, 5, and 7 in ankylotic stapes footplates with different histopathologies and in controls.

\begin{tabular}{lllll}
\hline $\begin{array}{l}\text { Histopathology } \\
\text { (H\&E) }(n=108)\end{array}$ & BMP2 & BMP4 & BMP5 & BMP7 \\
\hline $\begin{array}{l}\text { Otosclerosis }(n=51) \\
\text { Active focus }(n=39)\end{array}$ & +++ & ++++ & +++ & +++ \\
$\begin{array}{l}\text { Inactive focus } \\
(n=12)\end{array}$ & - & + & - & - \\
$\begin{array}{l}\text { Non-otosclerotic } \\
\text { stapes fixation } \\
(n=16)\end{array}$ & & & & \\
$\begin{array}{l}\text { Annular calcification } \\
(n=16)\end{array}$ & - & - & - & - \\
Controls $(n=41)$ & & & & \\
$\begin{array}{l}\text { Cortical bone } \\
(n=35)\end{array}$ & - & - & - & - \\
Incus $(n=6)$ & - & - & - & - \\
\hline
\end{tabular}

Subjective intensity assessment of immunofluorescence assays (IFAs) by the analysis of 10 centrally focused magnification fields ( $\times 40)$ : +, weak annular immunoreactivity; ++ , weak homogeneous immunoreactivity; +++ , strong homogeneous or weak granular immunoreactivity; ++++ , strong confluent and robust granular reaction.

indicating active bone remodeling and new bone formation within the lesion (Figure 1). The BMP immunoreactivity varied between different parts of the stapes samples. The superstructures were negative for BMPs, while the footplates containing the lesion and the hyaline cartilage layer of the vestibular surface displayed very similar expression patterns. The optimal exposure time was $43 \mathrm{~ms}$ for BMP-expressing otosclerotic specimens that were strongly positive by IFA. In BMPnegative samples (non-otosclerotic stapes footplates, control bone specimens), exposure time was extended until $77 \mathrm{~ms}$ to avoid false-negative results. In contrast, in inactive otosclerotic foci, no significant BMP expression could be demonstrated on the surface of cellular remnants (Figure 2). In these cases, expression levels and patterns of BMPs were strongly associated with the histopathological activity of otosclerosis (Table I). Nonotosclerotic stapes fixations displayed negative immunoreactions for BMPs (Figure 3, Table I). Cortical bones and incus specimens applied as negative controls for stapes fixation displayed negative BMP-specific immunoreactions (Figure 4, Table I). Finally, expression of different types of BMP was strongly associated with the histologic diagnosis of otosclerosis (Table I).

\section{Discussion}

Although several disease-associated monogenic loci have been mapped and linked to otosclerosis, experiments to identify causative genes and their proteins have been unsuccessful $[1,6]$. In addition, candidate gene association studies on COL1A1, COL1A2, and RELN have generated conflicting results $[7,11]$. A notable difference with our study is that diagnosis of otosclerosis was based on the medical history and audiometric findings of patients with stapes fixation without histopathological analysis of the surgically removed stapes footplates $[6,9,11]$. It might be explained by the limitations of stapedotomy, which is not a suitable method to obtain stapes footplate fragments containing the fixing lesions.

This study aimed to carry out precise immunohistological assessement of BMP expression in otosclerotic and non-otosclerotic stapes footplates compared to bone-specific controls. The results shown in this report are of value, since only three studies have reported a potential association between increased BMP expression and otosclerosis $[8,12,16]$. Furthermore, we confirmed the observations reported by Lehnerdt et al. that suspected BMP2, 4, and 7 as real causative agents in the pathogenesis of otosclerosis [8]. According to our current knowledge, this is the first study in the literature to evaluate the role of BMP5 in the pathogenesis of otosclerosis. The present study indirectly supports the proposition that increased expression of BMP2, BMP4, BMP5, and $\mathrm{BMP} 7$, four members of the TGF- $\beta$ superfamily, also plays a role in the otosclerotic bone remodeling disorder.

It has been suggested that different types of BMP and BMPRs may be involved in the pathologically increased bone turnover observed in otosclerosis $[8,12,16]$. As pioneers, Lehnerdt et al. have performed immunohistochemical analysis of BMPs and BMPRs in otosclerotic stapes footplates [8]. Significantly increased BMP2, BMP4, and BMP7 expression was demonstrated within the otosclerotic foci compared with normal and non-otosclerotic bone specimens [8]. Among BMPRs, BMPR-1B and BMPR-2 showed increased immunoreactivity, whereas BMPR-1A always exerted negative reaction [12]. Thus, the effects of BMPs may be mediated through type $1 \mathrm{~B}$ and type $2 \mathrm{BMPRs}$ in otosclerosis [12]. Later, the same group carried out immunofluorescent typing of different BMP isoforms in active otosclerotic tissues. Again, consequent expressions of BMP2, BMP4, and BMP7 were demonstrated [12]. Schrauwen et al. have reported the potential role of various $\mathrm{BMP}$ isoforms in the risk of otosclerosis [16]. There was a significant association between clinical otosclerosis and the increased expression of BMP2 and BMP4 [16]. Authors have reported two otosclerosis-associated single nucleotide polymorphisms (SNPs) in the genes of BMP2 and BMP4 [16]. 


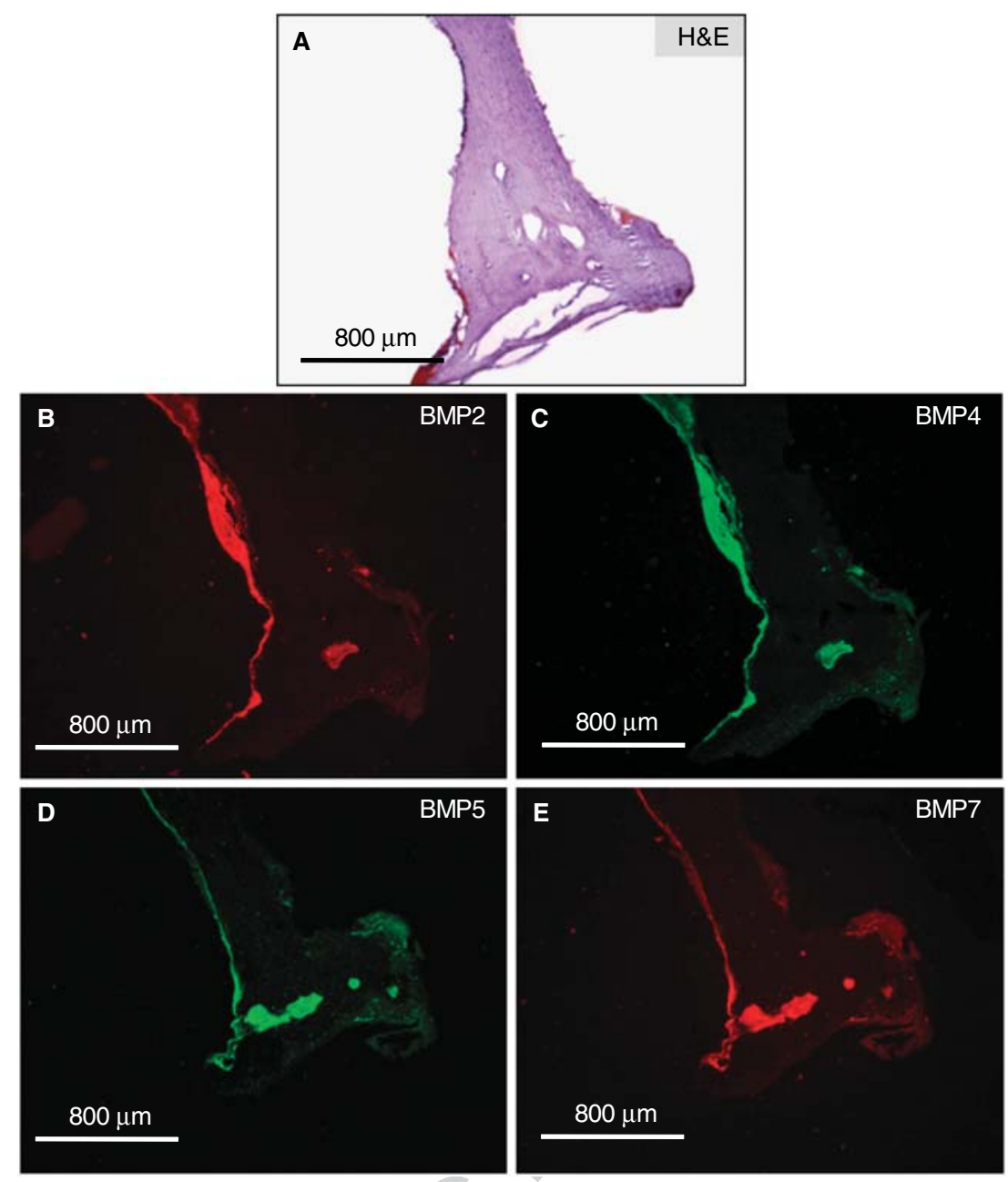

Figure 3. Immunofluorescent demonstration of BMP expression in non-otosclerotic stapes fixation. (A) Non-otosclerotic, annular calcification of the stapes footplate with hypocellular and angled anterior pole (H\&E, right ear). The osteocytes show negative immunoreactions for BMP2 (B), BMP4 (C), BMP5 (D), and BMP7 (E).

The first non-coding SNP, rs3178250 (BMP2), was located in a 3' untranslated region, while the second, rs17563 (BMP4), led to amino acid exchange in BMP4 that was strongly associated with otosclerosis [16]. These observations are reasonable to assume, since both BMPs and TGF- $\beta$ are key regulators of new bone formation. In addition, there are several interconnected events in the BMP and TGF- $\beta$ pathways that result in inverse interactions between the two protein groups $[17,18]$.

In the embryonic phase and in the early postnatal period, a regular enchondral ossification develops in the otic capsule and in the stapes footplate, which is completed after 1 year [1]. Osteoblastic and osteoclastic activity, normally associated with bone turnover, is rarely, if ever, seen in the adult otic capsule $[1,2]$. There is a potential explanation for this phenomenon: low-grade bone turnover protects the membranous labyrinth and the organ of Corti from serious biomechanical changes during life [1,2]. The otic capsule is formed by three bone-like layers, i.e. the endosteal layer next to the perilymphatic space, the interosseal globules (globuli inerossei), which are embryonic cartilage remnants, and the periosteal layer [1]. The enchondral layer of the otic capsule contains peculiar, embryonic chondrocytes, which persist throughout life $[1,19]$. Similar cells can be found in the vestibular layer of the stapes footplate. It has been reported that these remnants may be the sites of the earliest otosclerotic foci $[1,19]$. However, these cells disappear from otosclerotic foci and are replaced by pre-osteoclasts and active osteoclasts [19]. In contrast to normal adult otic capsule, osteoclasts and osteoblasts of otosclerotic foci express a special embryonic glycoprotein (CD51/61 - osteoclast functional antigen) [19]. In addition, increased expression of BMPs, TGF- $\beta$, tumor necrosis factor (TNF)- $\alpha$, and apoptosis inhibitors (hCIAP1/2) also supports 

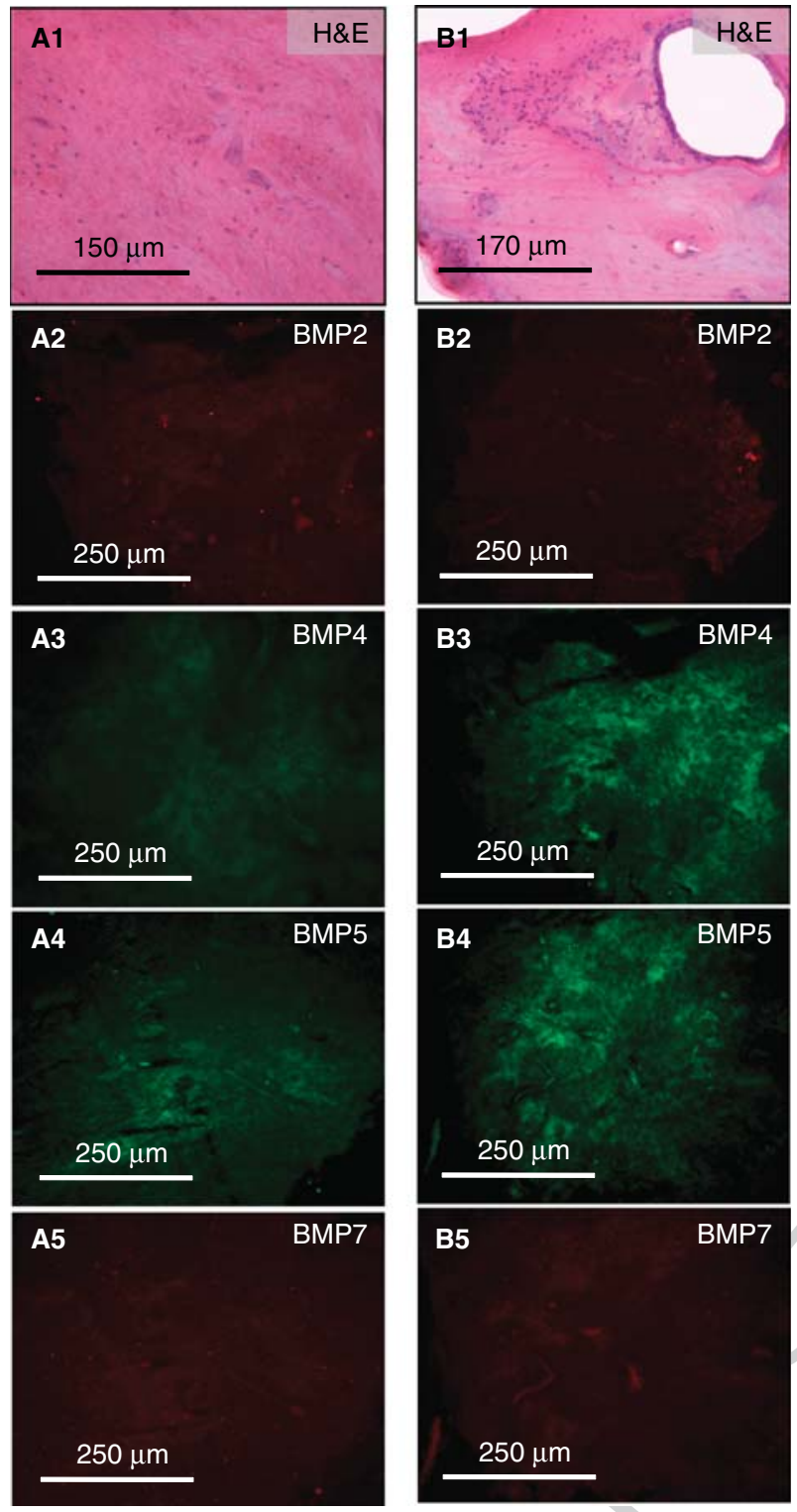

Figure 4. Immunofluorescent demonstration of BMP expression in bone-specific controls. (A1) Body of the incus that represents a normal structure of cement lines and blood vessels (H\&E, left ear). The osteocytes represent negative immunoreactions for BMP2 (A2), BMP4 (A3), BMP5 (A4), and BMP7 (A5). (B1) Cortical bone fragment harvested from the posterior wall of the external auditory canal (H\&E, left ear). The osteocytes do not display detectable expression of BMP2 (B2), BMP4 (B3), BMP5 (B4), or BMP7 (B5).

the embryonic chondrocyte reactivation hypothesis in otosclerosis $[1,8,9,20]$. According to previous reports, chondroblasts and osteoblasts might be reactivated and transformed into osteoclasts due to the inflammatory response induced by measles virus infection $[5,19,20]$.

In conclusion, otosclerosis can be considered as a complex bone remodeling disorder that is influenced by genetic, environmental, and metabolic factors.
According to current and previous results, it can be suggested that different types of BMPs may play a significant role in the early molecular events of active otosclerosis. In the light of this association, further studies are necessary to determine the role of BMP in the pathophysiology of otosclerosis to find the most promising medical (biological) treatment for early active stages.

\section{Acknowledgments}

This work was supported by grants from the Hungarian Scientific Research Fund (OTKA PD75371, K81480) and Mecenatúra Fund of University of Debrecen (DEOEC Mec 17/2008).

Declaration of interest: The authors report no conflicts of interest. The authors alone are responsible for the content and writing of the paper.

\section{References}

[1] Chole RA, McKenna M. Pathophysiology of otosclerosis. Otol Neurotol 2001;22:249-57.

[2] Stankovic KM, Adachi O, Tsuji K, Kristiansen AG, Adams JC, Rosen V, et al. Differences in gene expression between the otic capsule and other bones. Hear Res 2010; 265:83-9.

[3] Declau F, Van Spaendonck M, Timmermans JP, Michaels L, Liang J, Qiu JP, et al. Prevalence of otosclerosis in an unselected series of temporal bones. Otol Neurotol 2001;22:596-602.

[4] Karosi T, Csomor P, Petkó M, Liktor B, Szabó LZ, Pytel J, et al. Histopathology of nonotosclerotic stapes fixations. Otol Neurotol 2009;30:1058-66.

[5] McKenna MJ, Mills BG, Galey FR, Linthicum FH Jr. Filamentous structures morphologically similar to viral nucleocapsids in otosclerotic lesions in two patients. Am J Otol 1986;7:25-8.

[6] Van Den Bogaert K, Govaerts PJ, De Leenheer EM, Schatteman I, Verstreken M, Chen W, et al. Otosclerosis: a genetically heterogeneous disease involving at least three different genes. Bone 2002;30:624-30.

[7] McKenna MJ, Kristiansen AG, Bartley ML, Rogus JJ, Haines JL. Association of COL1A1 and otosclerosis: evidence for a shared genetic etiology with mild osteogenesis imperfecta. Am J Otol 1998;19:604-10.

[8] Lehnerdt G, Unkel C, Metz KA, Jahnke K, Neumann A. Immunohistochemical evidence of $\mathrm{BMP}-2,-4$ and -7 activity in otospongiosis. Acta Otolaryngol 2008;128:13-17.

[9] Thys M, Schrauwen I, Vanderstraeten K, Janssens $K$, Dieltjens N, Van Den Bogaert K, et al. The coding polymorphism T263I in TGF-beta1 is associated with otosclerosis in two independent populations. Hum Mol Genet 2007; 16:2021-30.

[10] Karosi T, Szalmás A, Csomor P, Kónya J, Petkó M, Sziklai I. Disease-associated novel CD46 splicing variants and pathologic bone remodeling in otosclerosis. Laryngoscope 2008; 118:1669-76. 
[11] Schrauwen I, Ealy M, Huentelman MJ, Thys M, Homer N, Vanderstraeten $\mathrm{K}$, et al. A genome-wide analysis identifies genetic variants in the RELN gene associated with otosclerosis. Am J Hum Genet 2009;84:328-38.

[12] Lehnerdt G, Metz KA, Trellakis S, Jahnke K, Neumann A. Signaling by way of type IB and II bone morphogenetic protein receptors regulates bone formation in otospongiosis. Laryngoscope 2007;117:812-16.

[13] de Gorter DJ, van Dinther M, Korchynskyi O, Ten Dijke P. Biphasic effects of transforming growth factor $\beta$ on bone morphogenetic protein-induced osteoblast differentiation. J Bone Miner Res 2011;26:1178-87.

[14] Liu W, Butts S, Kim H, Frenz DA. Negative regulation of otic capsule chondrogenesis: it can make you Smad. Ann N Y Acad Sci 2007;1116:141-8.

[15] Brown DJ, Kim TB, Petty EM, Downs CA, Martin DM, Strouse PJ, et al. Autosomal dominant stapes ankylosis with broad thumbs and toes, hyperopia, and skeletal anomalies is caused by heterozygous nonsense and frameshift mutations in NOG, the gene encoding noggin. Am J Hum Genet 2002; $71: 618-24$.
[16] Schrauwen I, Thys M, Vanderstraeten K, Fransen E, Dieltjens N, Huyghe JR, et al. Association of bone morphogenetic proteins with otosclerosis. J Bone Miner Res 2008;23: 507-16.

[17] Harris SE, Bonewald LF, Harris MA, Sabatini M, Dallas S, Feng JQ, et al. Effects of transforming growth factor beta on bone nodule formation and expression of bone morphogenetic protein 2, osteocalcin, osteopontin, alkaline phosphatase, and type I collagen mRNA in long-term cultures of fetal rat calvarial osteoblasts. J Bone Miner Res 1994;9:855-63.

[18] Zheng MH, Wood DJ, Wysocki S, Papadimitriou JM, Wang EA. Recombinant human bone morphogenetic protein-2 enhances expression of interleukin- 6 and transforming growth factor-beta 1 genes in normal human osteoblast-like cells. J Cell Physiol 1994;159:76-82.

[19] Karosi T, Jókay I, Kónya J, Petkó M, Szabó LZ, Pytel J, et al. Activated osteoclasts with CD51/61 expression in otosclerosis. Laryngoscope 2006;116:1478-84.

[20] Csomor P, Sziklai I, Liktor B, Szabó L, Pytel J, Jóri J, et al. Otosclerosis: disturbed balance between cell survival and apoptosis. Otol Neurotol 2010;31:867-74. 